\title{
Generalised Lambda Distributions by Method of Moments and Maximum Likelihood using the JSE-ASI Returns
}

\author{
Peterson Owusu Junior (Corresponding author) \\ Institute of Distance Learning (IDL) \\ Kwame Nkrumah University of Science \& Technology (KNUST) \\ PMB Kumasi, Ghana \\ Tel: 233-260-006-397_E-mail:bethpeniel@gmail.com \\ Carl H. Korkpoe \\ Department of Computer Science, School of Physical Sciences \\ University of Cape Coast, PMB Cape Coast, Ghana \\ Tel: 233-543-179-583Ｅ-mail: ckorkpoe@ucc.edu.gh
}

Received: Feb. 10, 2017 Accepted: April 5, $2017 \quad$ Published: June 1, 2017

doi:10.5296/ajfa.v9i1.10913 URL: http://dx.doi.org/10.5296/ajfa.v9i1.10913

\begin{abstract}
The four-parameter generalised lambda distribution provides the flexibility required to describe the key moments of any distribution as compared with the normal distribution which characterises the distribution with only two moments. As markets have increasingly become nervous, the inadequacies of the normal distribution in capturing correctly the tail events and describing fully the entire distribution of market returns have been laid bare. The focus of this paper is to compare the generalised method of moments (GMM) and maximum likelihood essential estimates (MLE) methods as subsets of the GLD for a better fit of JSE All Share Index returns data. We have demonstrated that the appropriate method of the GLD to completely describe the measures of central tendency and dispersion by additionally capturing the risk dimensions of skewness and kurtosis of the return distribution is the Generalised Method of Moments (GMM) with the Kolmogorov-Smirnoff Distance good-of-fit statistics and the quantile-quantile graph. These measures are very important to any investor in the equity markets.
\end{abstract}


Keywords: generalised hyperbolic distribution (GHD), generalised lambda distribution (GLD), modified Bessel function, MLE, GMM 


\section{Introduction}

Risk is important to investors for any level of market return. In frontier markets with short histories, long historical data may be lacking for modeling purposes. Market analysts are also absent in most cases to follow usually thinly traded equities to serve the investor community. This makes it extra challenging for investors to assess the risk-return characteristics of their portfolios in such markets.

We seek to ameliorate this challenge by providing a flexible four-parameter distribution model; the Generalised Lambda Distribution (GLD) to model the characteristics of market returns and the tails risk essential to guide market participants in forming efficient portfolios. In the process of comparing the better fit between the GMM and MLE under GLD we show the performance of our models by comparing them to the two-parameter normal distribution; a popular but rather discredited model in risk modeling of equity returns (see Fama \& MacBeth, 1973; Giot \& Laurent, 2004; Hull \& White, 1998).

Modeling market returns by using the normal distribution does not provide the flexibility needed as compared to, for instance, the four-parameter generalised lambda distribution. To capture the stylised facts of a security or a return series there arises the need to model the returns in their entirety. Value-at-Risk (VaR) vis-a-vis Expected Shortfall (ES), as good examples, requires us to capture the risk properties of a return using only the quantile values in the left tail of the distribution.

The need to model just the tail behaviour of the losses in assets returns is a matter of empirical concern. But is it well known that the normal distribution is a poor model for stock returns (Eberlein \& Keller, 1995). The classes of generalised hyperbolic distribution (GHD) have the capabilities of mirroring both tail behaviour and asymmetries of returns which is a desired property of distributions in finance. Besides the normal distribution many models have been investigated (see Eberlein \& Keller, 1995) but it was the class of hyperbolic distributions which turned out to be an excellent candidate to provide a more realistic model. In this paper we model the Johannesburg Stock Exchange All Share Index (JSE - ASI) with the generalised lambda distribution (GLD) using the generalised method of moments (GMM) and the maximum likelihood estimation (MLE) approaches to establish the better fit for the data.

Except for Corrado (2001), Tarsitano (2004), Chalabi, Scott, \& Wuertz (2012), and Pfaff, (2013) there has been little application of GHD; especially of the generalised lambda distribution (GLD) to data. Pfaff (2013) for instance, applies the GHD and its special cases, namely; hyperbolic distribution (HYP), normal inverse Gaussian distribution (NIG), and generalised lambda distribution (GLD) to financial market data; the Hewlett-Packard (HWP), to draw useful insights.

The shape range of the GLD family, for instance, is so large that it can accommodate almost any financial time series especially useful in the estimation of risk measures where the choice of distribution is crucial for accuracy (Chalabi et al., 2012). His work also showed that these multi-parameter distributions provide a good description of the distribution of equity returns 
for the DOW. We have, however, not seen these distributions in empirical work in emerging and frontier markets. Given investors perception that these two markets are risky (see Claessens, Dasgupta, \& Glen, 1995; Errunza \& Padmanabhan, 1988; Hassan, Maroney, El-Sady, \& Telfah, 2003; Hearn, Piesse, \& Strange, 2010); the need arises to establish risk-return characteristics in equity and indeed related securities in these markets on a sound theoretical footing. In this paper, therefore, we find the better fit for log returns of the Johannesburg Stock Exchange All Share Index (JSE-ASI) between the GMM and MLE methods.

\section{Theoretical Framework}

\subsection{Generalised Hyperbolic Distributions (GHD)}

Hyperbolic distributions are characterised by their log-density being a hyperbola unlike normal distributions whose log-density is a parabola. One can then expect to obtain a reasonable alternative for heavy tail distribution (Eberlein \& Keller, 1995). The parameterised hyperbolic density function can be given as

$$
\operatorname{hyp}(x)=\frac{\sqrt{\alpha^{2}-\beta^{2}}}{2 \alpha \delta \mathrm{K}_{\mathrm{I}}\left(\delta \sqrt{\alpha^{2}-\beta^{2}}\right)} \exp \left(-\alpha \sqrt{\delta^{2}+(x-\mu)^{2}}+\beta(x-\mu)\right)
$$

where $\mathrm{K}$ is the modified Bessel function of the third kind with index I. $\alpha$ and $\beta$ with $\alpha>0$ and $0 \leq|\beta|<\alpha$ determine the shape of the distribution, while $\delta$ and $\mu$ are scale and location parameters respectively. Koudou \& Ley (2014) assert that in 1977 Barndorff-Nielsen and Halgreen showed that the generalised inverse Gaussian distribution (GIGD), sometimes called Halphen Type A distribution, with the following probability density function

$$
\operatorname{gig}(x ; \lambda, \chi, \psi)=\frac{(\psi / \chi)^{\lambda / 2}}{2 \mathrm{~K}_{\lambda}(\sqrt{\chi \psi})} x^{\lambda-1} \exp \left\{-\frac{1}{2}\left(\chi x^{-1}+\psi x\right)\right\}, \quad(x>0)
$$

has the property of infinite divisibility which is used in the representation of hyperbolic distributions as a mixture of normals. Any mixture of the r-dimensional normal distributions $N_{r}(\xi, \Sigma)$ determined by setting $\xi=\mu+\sigma^{2} \beta \Delta$ and $\Sigma=\sigma^{2} \Delta$. This class of mixtures includes the r-dimensional hyperbolic distribution

$$
a(\alpha, \beta, \delta, \Delta) \exp \left\{-x \sqrt{\delta^{2}+(v-\mu) \Delta^{-1}(v-\mu)^{\prime}}+\beta((v-\mu))\right\}
$$

where $\Delta$ is a positive definite $r \times r$ matrix with determinant $|\Delta|=1$ and setting $\kappa=$ $\sqrt{\alpha^{2}-\beta \Delta \beta^{\prime}}$, the norming constant is given by

$$
a(\alpha, \beta, \delta, \Delta)=\frac{1}{(2 \pi)^{(r-1) / 2}} \cdot \frac{\kappa^{(r+1) / 2}}{2 \alpha \delta^{(r+1) / 2} \mathrm{~K}_{(r+1) / 2}(\delta \kappa)}
$$


was introduced by the same authors a year earlier (Barndorff-Nielsen, Blaesild, \& Halgreen, 1978; O. Barndorff-Nielsen \& Halgreen, 1977).

Based on the daily prices of the 30 DAX shares over a three-year period Eberlein \& Keller (1995) investigated the distributional form of compound returns after performing a number of statistical tests to the logical conclusion that some standard assumptions could not be justified. Hence, they introduced the class of hyperbolic distributions which can be fitted to empirical returns with high accuracy. This was the application of GHD to the increments of financial market price processes probably first proposed by Eberlein and Keller in 1995 (i.e. the returns resulting from geometric Brownian motion are increments of a Brownian motion process, thus are independent and normally distributed). Again tests applied to real data in this way demonstrate failure of the normality assumption.

The popularity of the class of GHDs in finance was pioneered in seminal papers in the late 1990s (see (Barndorff-Nielsen \& Shephard, 1998; Barndorff-Nielsen \& Halgreen, 1977; Eberlein, Keller, \& Prause, 1998; Prause, 1997, 1999). However the application of hyperbolic distributions in fields other than finance is not limited. The motivation for Barndorff-Nielsen \& Halgreen (1977) to introduce the hyperbolic distributions was the observation by Bagnold (1941) that the logarithm of the density function of distribution of the logarithm of the grain size in natural sand deposits looks more like a hyperbola than a parabola (Sørensen, 2006).

\subsection{Generalised Lambda Distribution (GLD)}

The four-parameter (quantile) GLD family is known for its high flexibility, producing distributions with a range of different shapes. To start with (Ramberg \& Schmeister, 1974) show six regions in which the shape parameters can lie for which the shapes of the GLD are similar, as shown in Figure 1; the figure is as given in King \& MacGillivray (1999) with notation introduced by Ramberg, Dudewicz, Tadikamalla, \& Mykytka (1979), Chalabi et al. (2012), and Chalabi, Scott, \& Würtz (2010). The modern Freimer, Kollia, Mudholkar, \& Lin (1988) - FMKL GLD (2.6) places the only restriction of $\lambda_{4}>0$. The fundamental motivation for the development of FMKL GLD is that the distribution is defined over all $\lambda_{3}$ and $\lambda_{4}$ (Su, 2007b).

The quantile probability density function of the GLD (henceforth referred to as RS GLD) is given as the inverse distribution function of Tukey's lambda distribution (TLD)

$$
F^{-1}(\rho \mid \lambda)=F^{-1}\left(\rho \mid \lambda_{1}, \lambda_{2}, \lambda_{3}, \lambda_{4}\right)=\lambda_{1}+\frac{\rho^{\lambda_{3}}-(1-\rho)^{\lambda_{4}}}{\lambda_{2}}
$$

where $\rho$ are the probabilities, $\rho \in[0,1], \lambda_{1}, \lambda_{2}$ are the location and scale parameters, and $\lambda_{3}, \lambda_{4}$ are the shape parameters jointly related to the strengths of the lower and upper tails, respectively. The original one-parameter TLD results in the limiting case $\lambda_{1}=0$ and $\lambda_{2}=\lambda_{3}=\lambda_{4}=\lambda$ (Chalabi et al., 2010).

The FMKL GLD can be written as 


$$
F^{-1}(\rho \mid \lambda)=F^{-1}\left(\rho \mid \lambda_{1}, \lambda_{2}, \lambda_{3}, \lambda_{4}\right)=\lambda_{1}+\frac{\frac{\rho^{\lambda_{3}-1}}{\lambda_{3}}-\frac{(1-\rho)^{\lambda_{4}-1}}{\lambda_{4}}}{\lambda_{2}}
$$

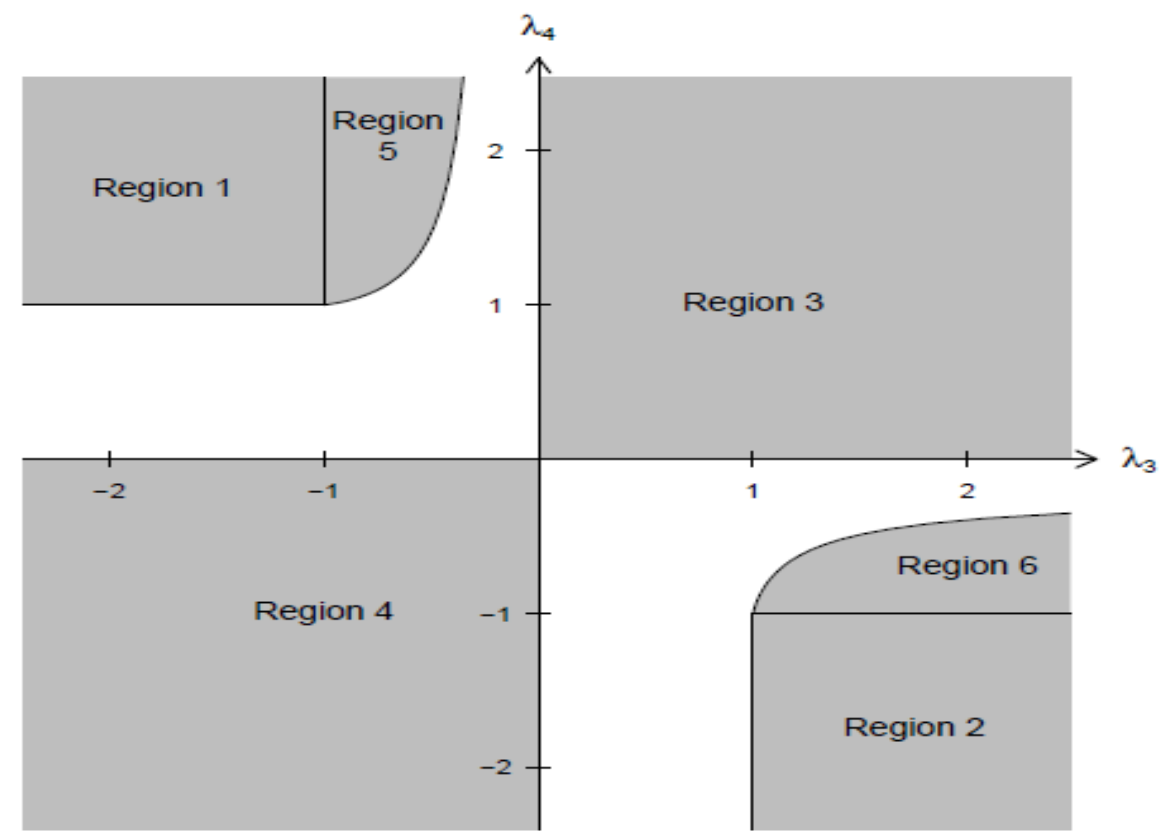

Figure 1. Graph of the parameters regions of the GLD in the $\lambda_{3}$ vs. $\lambda_{4}$ space that produce proper statistical distributions. Source: (Chalabi et al., 2010)

The parameter constellations that fall in the third quadrant imply skewed and heavy-tailed distributions; that is a nice characteristic of stylised facts about financial return series giving more credence to the use of non-normal distributions, in this case GLD to capture this behaviour (Pfaff, 2013).

The various methods for estimation of the optimal values for the parameter vector $\lambda$ in the literature include, among others (1) moment-matching approach by Ramberg \& Schmeister (1974) and Ramberg et al. (1979), (2) percentile-based approach by Karian \& Dudewicz (1999), (3) histogram-based approach by Su (2005), (4) goodness-of-fit approach by Owen (1988), (5) maximum likelihood (ML) and maximum spacing approaches by Cheng \& Amin (1983) and Ranneby (1984) and (6) least squares (LS)-approach (see Karvanen \& Nuutinen, 2008; Öztürk \& Dale, 1982, 1985).

$\mathrm{Su}$ (2007a) discussed two general approaches to fitting generalised lambda distributions to data; using the discretised method and maximum likelihood estimation (or an approach that aims to provide a definite fit to the data set such as maximising the goodness of fit) with his own authored GLDEX package in $\mathrm{R}$ ( $\mathrm{R}$ Core Team, 2016). However, the maximum likelihood estimation is preferred to the former not just for its efficiency but also likely to produce GLD with closer first four moments to the data set (see Su, 2005, 2007a). 
Stemming from an extensive investigation by Karian \& Dudewicz (2000), King \& MacGillivray (1999), Ramberg \& Schmeister (1974), Ramberg et al. (1979), etc. on the symmetry distribution version of the GLD given for $\lambda_{3}=\lambda_{4}$, not all parameter combinations yield valid density functions (Pfaff, 2013). The probability density function of the GLD at $x=F^{-1}(\rho \mid \lambda)$ is given by

$$
f(x)=f\left(F^{-1}(\rho \mid \lambda)\right)=\frac{\lambda_{2}}{\lambda_{3} \rho^{\lambda_{3}-1}-\lambda_{4}(1-\rho)^{\lambda_{4}-1}}
$$

Valid parameter combinations of $\lambda$ must yield (2.8) and (2.9):

$$
\begin{gathered}
f(x) \geq 0 \\
\int f(x) d x=1
\end{gathered}
$$

for $f(x)$ in (2.7) to qualify as density function (Pfaff, 2013).

This is going to be the only mention of symmetry in this paper since the focus is fitting the GLD to financial return series. The limitation pointed out here does not affect the superiority of the GLD over normal distribution, at least, in fitting the JSE-ASI.

One advantage of the GLD is that the computation of VaR and ES can easily be achieved after a return series has been fitted herewith; expressed as positive numbers of returns and not for losses (Pfaff, 2013).

Hyperbolic distributions are characterised by their log-density being a hyperbola unlike normal distributions whose log-density is a parabola. One can then expect to obtain a reasonable alternative for heavy tail distribution (Eberlein \& Keller, 1995). The parameterised hyperbolic density function can be given as

$$
\text { hyp }(x)=\frac{\sqrt{\alpha^{2}-\beta^{2}}}{2 \alpha \delta \mathrm{K}_{\mathrm{I}}\left(\delta \sqrt{\alpha^{2}-\beta^{2}}\right)} \exp \left(-\alpha \sqrt{\delta^{2}+(x-\mu)^{2}}+\beta(x-\mu)\right)
$$

where $\mathrm{K}$ is the modified Bessel function of the third kind with index I. $\alpha$ and $\beta$ with $\alpha>0$ and $0 \leq|\beta|<\alpha$ determine the shape of the distribution, while $\delta$ and $\mu$ are scale and location parameters respectively. Koudou \& Ley (2014) assert that in 1977 Barndorff-Nielsen and Halgreen showed that the generalised inverse Gaussian distribution (GIGD), sometimes called Halphen Type A distribution, with the following probability density function

$$
\operatorname{gig}(x ; \lambda, \chi, \psi)=\frac{(\psi / \chi)^{\lambda / 2}}{2 \mathrm{~K}_{\lambda}(\sqrt{\chi \psi})} x^{\lambda-1} \exp \left\{-\frac{1}{2}\left(\chi x^{-1}+\psi x\right)\right\}, \quad(x>0)
$$

has the property of infinite divisibility which is used in the representation of hyperbolic distributions as a mixture of normals. Any mixture of the r-dimensional normal distributions $N_{r}(\xi, \Sigma)$ determined by setting $\xi=\mu+\sigma^{2} \beta \Delta$ and $\Sigma=\sigma^{2} \Delta$. This class of mixtures includes the r-dimensional hyperbolic distribution 


$$
a(\alpha, \beta, \delta, \Delta) \exp \left\{-x \sqrt{\delta^{2}+(v-\mu) \Delta^{-1}(v-\mu)^{\prime}}+\beta((v-\mu))\right\}
$$

where $\Delta$ is a positive definite $r \times r$ matrix with determinant $|\Delta|=1$ and setting $\kappa=$ $\sqrt{\alpha^{2}-\beta \Delta \beta^{\prime}}$, the norming constant is given by

$$
a(\alpha, \beta, \delta, \Delta)=\frac{1}{(2 \pi)^{(r-1) / 2}} \cdot \frac{\kappa^{(r+1) / 2}}{2 \alpha \delta^{(r+1) / 2} \mathrm{~K}_{(r+1) / 2}(\delta \kappa)}
$$

was introduced by the same authors a year earlier (Barndorff-Nielsen, Blaesild, \& Halgreen, 1978; O. Barndorff-Nielsen \& Halgreen, 1977).

\section{Methodology}

In comparing MLE and GMM we are embarking on a journey of goodness of fit. Thus going through parameter estimation procedure to find the values of GMM and MLE that best fit the JSE-ASI data. The GMM and MLE have come head-to-head as alternatives to statistical estimation since over two decades after the former was introduced in 1894 by Karl Pearson. In recent years, however, financial econometricians have found working under assumed likelihood functions restrictive, and have suggested using a generalised version of Pearson's MM approach, commonly known as GMM estimation procedure as advocated by Bera \& Bilias (2002) and Hansen (1982).

Fisher (1922) asserts that the parameters of the MM are those of an infinite population of the specified type having the same moments as those calculated from the sample, its application has not been shown except in the case of the normal curve. To discover whether this approximation is good or bad he proposes an improvement, if possible, by requiring a more adequate criterion. By assessing the suggestions from Fisher (1922), Pearson (1936) found that the additional computing work after the determination by moments is "as long as, I think longer than, the first fitting by moments". The blunders in both MM and MLE are explored in the same paper thereof. Notwithstanding, GMM is seen, largely, as an applicable parameter estimation strategy which shells the classic method of moments, linear regression, and maximum likelihood (Chaussé, 2010, 2012).

\subsection{Generalised Method of Moments (GMM)}

Pearson (1894) introduced a formal approach to statistical estimation through his method of moments (MM) where estimators or parameters are derived from moment conditions estimating parameters of distributions using moment equations. As hinted by Pearson (1936) GMM estimation is often possible where a likelihood analysis is extremely difficult and Wooldridge (2001); that the MM applies in more complicated situations. The theory of GMM, restricted to large-sample comparisons helps with the use of two unbiased consistent method of moments estimators in a manner that minimises the asymptotic variance among method of moment's estimators (Wooldridge, 2001). 


\section{Macrothink}

The GMM is relatively advantageous for time series data. This comes as no surprise for the reason that Hansen (1982) introduced the GMM primarily with time series applications in mind, and it can be used to obtain parameter estimators that are consistent under weak distributional assumptions (Wooldridge, 2001). The method of moments consists of equating empirical moments with theoretical moments Focardi \& Fabozzi (2004) in order to get estimators of the parameters (Iacus, 2011).

Chaussé (2010) and Chaussé (2012) thus conclude; because GMM depends only on moment conditions, it is a reliable estimation procedure for many models in economics and finance. With the right moment conditions the GMM is more appropriate than maximum likelihood. In this paper we see how that plays out for our set of data; a corroboration.

Further, in finance, there is no satisfying parametric distribution which reproduces the properties of stock returns except that the family of stable distributions is a good candidate. In this regard only the densities of the normal, Cauchy, and Levy distributions have a closed form expression (Chaussé, 2010).

The mathematical narrative that follows is based on Chaussé (2010). We would want a vector of parameters $\theta_{0} \in \mathbb{R}^{p}$ based on $q \times 1$ vector of unconditional moments below:

$$
E\left[g\left(\theta_{0}, x_{i}\right)\right]=0
$$

where $x_{i}$ is vector of cross-sectional data, time series, or both. $\theta_{0}$ has to be the unique solution to (3.0) and be an element of a compact space for GMM to produce consistent estimates. Higher boundary conditions of $g\left(\theta_{0}, x_{i}\right)$ are also needed.

Given the linear model (3.1) in $Y(n \times 1)$ and $X(n \times 1)$ matrices:

$$
Y=X \beta+u
$$

The most common method of estimating the parameter $\hat{\beta}$ is least squares (LS) approach by solving $\min _{\beta}\|u\|^{2}$ hence the solution to the first order condition:

$$
\frac{1}{n} X^{\top} u(\beta)=0
$$

Equation (3.1) is the estimate of the moment condition of:

$$
E\left(X_{i}, u_{i}(\beta)\right)=0
$$

The same model can be estimated by ML in which case the moment condition becomes (3.4) below similar to $(3.8)$ : 


$$
E\left[\frac{d l_{i}(\beta)}{d \beta}\right]=0
$$

given that $l_{i}(\beta)$ is the density of $u_{i}$ (Baum, Schaffer, \& Stillman, 2003; Baum et al., 2003; Baum, Schaffer, Stillman, \& others, 2007; Hansen, 1982; Hansen, Heaton, \& Yaron, 1996; Stock, Wright, \& Yogo, 2002). A lot of estimation methods including LS, ML, or instrumental variables (IV) can also be seen as being based on such moment conditions as in (3.2), therefore, they are special cases of GMM (Chaussé, 2010, 2012).

The moment condition is obtained by equating the derivatives of a log-likelihood to zero that MLE can be given an MM interpretation as well; (see Chaussé, 2010, 2012) for the relationships between GMM and IV, generalised LS, and ML, and pseudo-ML. Generally, the moment conditions $E\left[g\left(\theta_{0}, x_{i}\right)\right]=0(3.0)$ is a vector of non-linear functions of the parameter $\theta_{0}$ with the number of unlimited by the same parameter (Chaussé, 2010, 2012). It is, therefore, a step in the right direction to estimate the parameters on GLD by GMM as well as MLE in this paper. In order to reduce the possibility of inefficiency there is the need to increase the number of instruments $q$ which is often greater than $p$ rendering (3.5) with no solution.

$$
\bar{g}(\theta)=\frac{1}{n} \sum_{i=1}^{n} g\left(\theta_{0}, x_{i}\right)=0
$$

Remedial methods for this situation are offered by Hansen (1982), with so-called two-stage GMM (2SGMM) in the original version of GMM, also Andrews (1991) and Newey \& West (1987) with a heteroscedasticity and autocorrelation consistent (HAC)-like matrix. Further improvement to the properties of 2SGMM by Hansen et al. (1996) are the iterative 2SGMM (IT2SGMM) and the continuous updated estimator.

Like the MLE, GMM estimators are easily consistent, however, efficiency bias depend on the choice of moment conditions (Chaussé, 2010). Though the distribution-free feature of GMM gives a very good appeal, it is as good as its moment conditions.

\subsection{Maximum Likelihood Estimation (MLE)}

Originally developed by R.A. Fisher in the 1920s, the principle of the MLE states that the desired probability distribution is the one that makes the observed data 'most likely', which means that one seeks the value of the parameter vector that maximises the likelihood function (3.1) where $w=\left(w_{1}, \ldots, w_{k}\right)$ is parameter vector defined on a multi-dimensional parameter space and $y=\left(y_{1}, \ldots, y_{n}\right)$ is a random sample data vector (Myung, 2003). It is a principle of statistical estimation which, given a parametric model such as the GLD, prescribes choosing those parameters that maximises the likelihood of the sample under the model; Focardi \& Fabozzi (2004) find this idea highly intuitive. 


\section{Macrothink}

Unlike least-squares estimation which is primarily a descriptive tool, MLE is a preferred method of parameter estimation in statistics and it's an indispensable tool for many statistical modelling techniques, in particular non-linear modeling with non-normal data (Myung, 2003).

Out of two possible general approaches to fitting GLD to data, according to Su (2007a); using the discretised method and a definite fit to the data set such as maximising the goodness of fit, the MLE is usually the preferred method (see King \& MacGillivray, 1999).

Let $f(y \mid w)$ denote the probability density function (PDF) that specifies the probability of observing vector $y$ given the parameter $w$. The likelihood function can be given as:

$$
L(w \mid y)=f(y \mid w)
$$

For computational convenience the MLE estimate is obtained by maximising the log-likelihood function Myung (2003) so that the product (3.2) is transformed into a sum. Since the logarithm is an increasing function, maximising the likelihood or the log-likelihood gives the same results

(Focardi \& Fabozzi, 2004). If the sample $y$ is IID then the likelihood is the product of individual likelihoods:

$$
L(w \mid y)=\prod_{i=1}^{n} f(y \mid w)
$$

Assuming the log-likelihood function $\ln L(w \mid y)$, is differentiable, if $w_{M L E}$ (MLE estimate) exists, it must satisfy the following partial differential equation called the likelihood equation:

$$
\frac{\partial \ln L(w \mid y)}{\partial w_{i}}=0
$$

Equation (3.3) is only a necessary condition for the existence of MLE estimates. A sufficient condition requires that $\ln L(w \mid y)$ is a maximum and not minimum. Hence the shape of the function be convex. Again from calculus (3.4) below must be true for $i=1,2, \ldots, k$.

$$
\frac{\partial^{2} \ln L(w \mid y)}{\partial w_{i}^{2}}<0
$$

For further reading see (Myung 2003; Iacus 2011).

In estimating the parameters of GLD the gld and GLDEX packages in R were employed. It is necessary to obtain quantiles, $\rho_{i}$ under the RS or FMKL for every observation $x_{i}$, for $i=1,2,3, \ldots, n$ under a set of initial values by solving (2.5) via Newton-Raphson method or numerically $(\mathrm{Su}, 2007 \mathrm{~b})$. By substituting $\rho_{i}$ 's obtained into the appropriate numerical $\log$ 
likelihood equations (3.9) and (3.10) below:

$$
\begin{gathered}
M L_{R S}=\sum_{i=1}^{n} \log \left[\frac{\lambda_{2}}{\lambda_{3} \rho_{i}^{\lambda_{3}-1}+\lambda_{4}\left(1-\rho_{i}\right)^{\lambda_{4}-1}}\right] \\
M L_{F M K L}=\sum_{i=1}^{n} \log \left[\frac{\lambda_{2}}{\rho_{i}^{\lambda_{3}-1}+\left(1-\rho_{i}\right)^{\lambda_{4}-1}}\right]
\end{gathered}
$$

The key is to maximise the likelihood in (3.5) and (3.6); this can be done through the Nelder-Simplex algorithm (Su, 2007b). The MLE estimates can be heavily biased but asymptotically consistent, efficient, and unbiased for adequately large sample size (Iacus, 2011).

\section{Data and Terminology}

The price series of the JSE-ASI is collected daily from January 02, 2003 to December 31, 2015 giving a total of three thousand two hundred and fifty-two data points. The log return series of the JSE - ASI was calculated as:

$$
r_{t}=\ln \left(1+R_{t}\right)=\ln \left(P_{t}\right)-\ln \left(P_{t-1}\right)
$$

where $r_{t}$ is the continuously compounded return (natural logarithm of the simple gross return of an asset). According to Tsay (2010), statistical properties of log returns are more tractable. For average investors, returns is a complete scale-free summary of investment prospect. Campbell et al. (1998) also showed that returns have more attractive statistical properties such as stationarity and ergodicity. A time series plot of the returns is show in Figure 2.

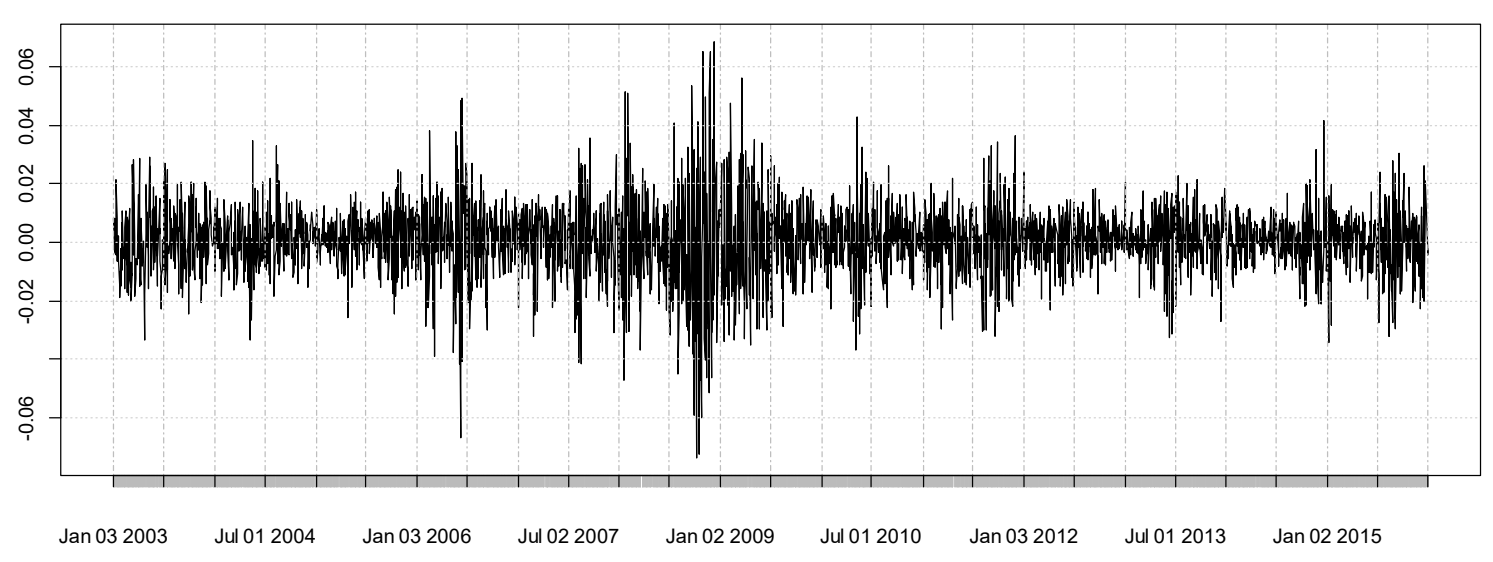

Figure 2. A time series plot of the JSE - ASI returns from January 2003 to January 2015 


\section{Macrothink}

The time series plot exhibits two regimes - 2003 to 2009 and 2010 to 2015 . In the first period the markets returns appeared more volatile particularly towards the end of the period in 2009 . This is contrast to the period 2010 to 2015 when the market showed moderate volatility. Such behaviour in financial assets in the markets has been observed by previous researchers (Assoe, 1998; Chkili \& Nguyen, 2014; Hamilton, 1989; M. R., 2001; van Norden \& Schaller, 1993).

Thus the statistical properties of the returns series will differ according to period. We therefore analysed the return series of the latter period of 2010 to 2015. Campbell, Lo, MacKinlay, \& Whitelaw (1998) discuss at length the problem associated with using long-term horizon data series. Among others they posit the breakdown of the asymptotic inferences from such data. Most likely that may be due to what Hamilton (1989) identified as regime switching in the literature. We believe our data window more reflects the recent developments in the underlying economy which are transmitted to the market.

\subsection{Testing for Classical Normality Assumptions}

A test of the classical assumptions of normality looks at the first four moments of the distribution. The skewness and kurtosis show slight deviations from normality. The curve of the distribution is skewed to the left with fat-tails as the results show in the Table 1.

Table 1. Moments of the JSE - ASI returns from January, 2003 to January 2015

\begin{tabular}{|l|r|r|r|}
\hline Mean & Variance & Skewness & Kurtosis \\
\hline 0.0005 & 0.00009 & -0.1701 & 1.4194 \\
\hline
\end{tabular}

A qualitative method for testing goodness of fit is quantile-quantile (QQ) plots juxtaposed with empirical densities (Eberlein \& Keller, 1995). The Q-Q plots alongside the histogram with the normal distribution curve of the log returns of the JSE-ASI provide an explicit empirical deviation from normality as shown in Figure 3. 

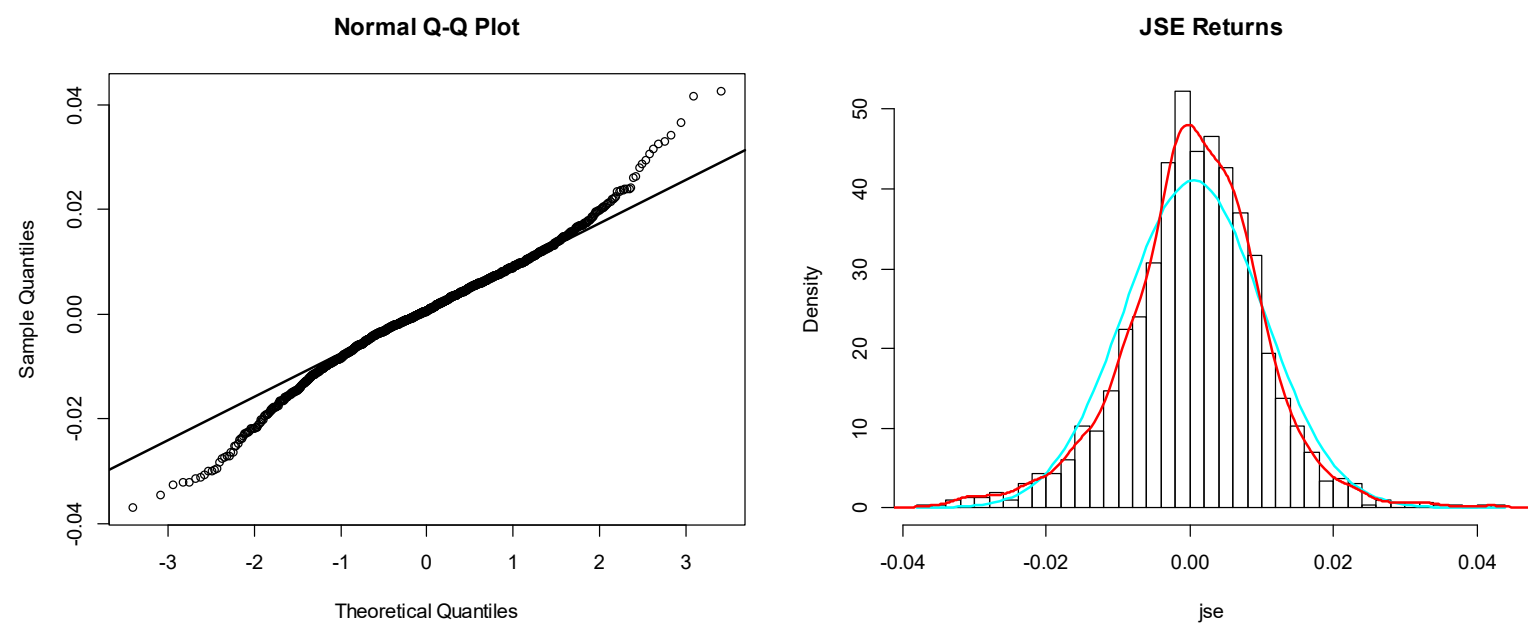

Figure 3. Q-Q plot and Normal distribution curve of the JSE - ASI returns from January, 2003 to January 2015

Finally in the Jarque-Bera and Cramer-von Mises normality tests; p-values respectively are nearly zero thus rejecting the null hypothesis of normality (Cromwell, Labys, \& Terraza, 1994; D'Agostino, 1986). We were, therefore, able to reach the conclusion that the returns series of the JSE returns were not normally distributed.

\subsection{Empirical Results from Methodology}

From the above series of tests it is obvious the return series of the JSE All Share Index distribution shows marked departures from normality. We thus use the Generalized Lambda Distribution (GLD) to fit the entire distribution - the GLD not only models the tail behaviour but also the entirety of the return distribution (Pfaff, 2013). We used our selected method of moments and maximum likelihood estimation respectively.

\subsection{Method of Moments}

The estimated parameters of the Generalized Lambda Distribution using the method of moments are shown in Table 2.

Table 2: The four parameters GLD obtained from the JSE - ASI returns from January, 2003 to January 2015

\begin{tabular}{|l|l|l|l|}
\hline $\boldsymbol{\lambda 1}$ & $\boldsymbol{\lambda 2}$ & $\boldsymbol{\lambda \mathbf { 3 }}$ & $\boldsymbol{\lambda \mathbf { 4 }}$ \\
\hline 0.072966 & 1.899254 & -0.0288 & 0.00986 \\
\hline
\end{tabular}

The parameters are the used to estimate the moments of the markets returns in Table 3. 


\section{Macrothink}

Table 3. The four moments of GMM obtained from the JSE - ASI returns from January, 2003 to January 2015

\begin{tabular}{|l|r|r|r|}
\hline Mean & Variance & Skewness & Kurtosis \\
\hline 0.0522 & 0.9453 & -0.1701 & 1.4193 \\
\hline
\end{tabular}

These values compared to the moments from the normal distribution are almost the same. However a test of Kolmogorov-Smirnoff Distance (D) recommended by Su (2007b), gave the distance $\mathrm{D}=0.97411$ with a $\mathrm{p}$-value of 0.1 . This test has the null hypothesis that the sample data is drawn from the same distribution as the fitted distribution. With a p-value of 0.1 , we fail reject the null hypothesis and conclude that the returns data came from the GLD using the method of moments.

\subsection{Maximum Likelihood Method}

Parametrising the GLD using the maximum likelihood method we get the values as shown in the Table 4.

Table 4. The four parameters of the parameterised GLD obtained from the JSE - ASI returns from January, 2003 to January 2015

\begin{tabular}{|l|l|l|l|}
\hline $\boldsymbol{\lambda 1}$ & $\boldsymbol{\lambda 2}$ & $\boldsymbol{\lambda} \mathbf{3}$ & $\boldsymbol{\lambda} \mathbf{4}$ \\
\hline 0.092051 & 2.037581 & -0.0832 & -0.01676 \\
\hline
\end{tabular}

Using these parameters to compute the moments of the distribution we get Table 5.

Table 5. The four moments of the parameterised GLD obtained from the JSE - ASI returns from January, 2003 to January 2015

\begin{tabular}{|l|l|l|l|}
\hline Mean & Variance & Skewness & Kurtosis \\
\hline 0.055878 & 0.957247 & -0.36028 & 2.520229 \\
\hline
\end{tabular}

A test of goodness-of-fit using the Kolmogorov-Smirnoff Distance test gives $\mathrm{D}=0.97185$ with a p-value of almost zero which is far the 5\% level of significance. Therefore, we reject the null hypothesis that the sample is drawn from the GLD using the maximum likelihood method. A graphical plot of the histograms for either method of estimation is shown in Figure 4 and Figure 5. 
Method of Moments Fit

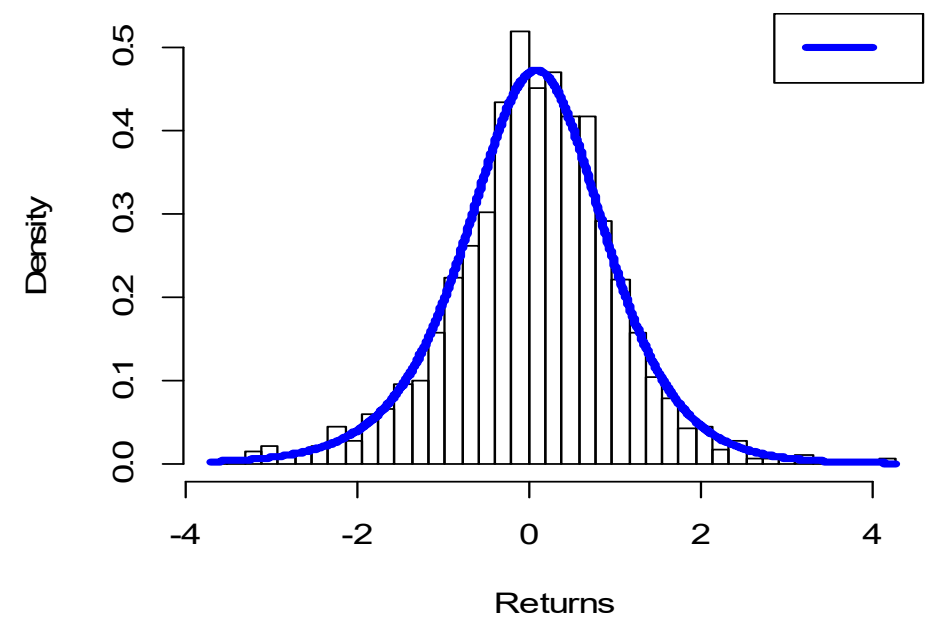

Figure 4. A histogram of the JSE - ASI returns from January, 2003 to January 2015 obtained by $\mathrm{MM}$

Method of Maximum Likelihood

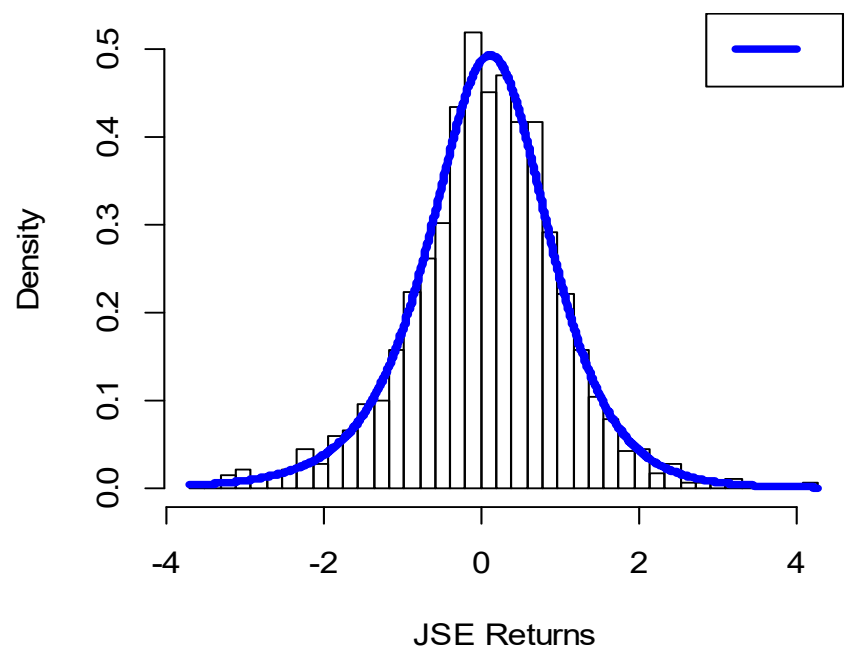

Figure 5. A histogram of the JSE - ASI returns from January, 2003 to January 2015 obtained by MLE

From Figure 4 and Figure 5 it is clear that using the GLD with the method of moments gave a histogram that closely described the entire distribution. Though the maximum likelihood method fairly described the data, it comes at a further computational effort making the method of moments more suitable for the estimation period.

\section{Conclusions}

Measuring volatility accurately has become the preoccupation of financial institutions as it determines their survival in increasingly turbulent markets. For tail risk measures like value-at-risk (VaR) and expected tail loss (ETL), the correct distribution of the data is a first 
step. Markets have swung from end to end on the volatility spectrum so violently that the normal distribution no longer accurately describes the entire distribution nor gives the correct quantiles in the tails for risk management purposes.

We have demonstrated the use of the generalized lambda distribution as an alternative using the method of moments and maximum likelihood estimates in modeling the return series of the JSE All Share index. Overall both the method of moments and maximum likelihood methods describe fairly accurately the distribution of the returns.

However, the method of moments with the Kolmogorov-Smirnoff Distance good-of-fit statistics and the quantile-quantile graph provides a better fit to the data. We have provided a basis for estimating the tail events useful in risk and portfolio management on the Johannesburg Stock Exchange.

\section{References}

Andrews, D. W. K. (1991). Heteroskedasticity and Autocorrelation Consistent Covariance Matrix Estimation. Econometrica, 59(3), 817-858. https://doi.org/10.2307/2938229

Assoe, K. (1998). Regime-Switching in Emerging Stock Market Returns (SSRN Scholarly Paper No. ID 2631519). Rochester, NY: Social Science Research Network. Retrieved from http://papers.ssrn.com/abstract $=2631519$

Bagnold, R. A. (1941). The physics of wind blown sand and desert dunes. Methuen, London, 265(10).

Barndorff-Nielsen, O., Blaesild, P., \& Halgreen, C. (1978). First hitting time models for the generalized inverse Gaussian distribution. Stochastic Processes and Their Applications, 7(1), 49-54. https://doi.org/10.1016/0304-4149(78)90036-4

Barndorff-Nielsen, O. E., \& Shephard, N. (1998). Aggregation and model construction for volatility models. Nuffield College. Retrieved from http://www.nuff.ox.ac.uk/economics/papers/1998/w7/ECON.PDF

Barndorff-Nielsen, O., \& Halgreen, C. (1977). Infinite divisibility of the hyperbolic and generalized inverse Gaussian distributions. Zeitschrift Für Wahrscheinlichkeitstheorie Und Verwandte Gebiete, 38(4), 309-311. https://doi.org/10.1007/BF00533162

Baum, C. F., Schaffer, M. E., \& Stillman, S. (2003). Instrumental variables and GMM: Estimation and testing. Stata Journal, 3(1), 1-31.

Baum, C. F., Schaffer, M. E., Stillman, S., \& others. (2007). Stata module for extended instrumental variables/2SLS, GMM and AC/HAC, LIML and k-class regression. Boston College Department of Economics, Statistical Software Components S, 425401, 2007.

Bera, A. K., \& Bilias, Y. (2002). The MM, ME, ML, EL, EF and GMM approaches to estimation: a synthesis. Journal of Econometrics, 107(1-2), 51-86. https://doi.org/10.1016/S0304-4076(01)00113-0

Campbell, J. Y., Lo, A. W., MacKinlay, A. C., \& Whitelaw, R. F. (1998). The econometrics of 
financial markets. Macroeconomic Dynamics, 2(04), 559-562.

Chalabi, Y., Scott, D. J., \& Wuertz, D. (2012). Flexible distribution modeling with the generalized lambda distribution. Retrieved from https://mpra.ub.uni-muenchen.de/43333/

Chalabi, Y., Scott, D. J., \& Würtz, D. (2010). The generalized lambda distribution as an alternative to model financial returns. Working paper, Eidgenössische Technische Hochschule and University of Auckland, Zurich and Auckland. Retrieved from https://www.rmetrics.org/sites/default/files/glambda_0.pdf

Chaussé, P. (2010). Computing Generalized Method of Moments and Generalized Empirical Likelihood with R. Journal of Statistical Software, 34(i11). Retrieved from https://ideas.repec.org/a/jss/jstsof/34i11.html

Chaussé, P. (2012). Generalized empirical likelihood for a continuum of moment conditions. Retrieved from http://www.archipel.uqam.ca/4653

Cheng, R. C. H., \& Amin, N. A. K. (1983). Estimating parameters in continuous univariate distributions with a shifted origin. Journal of the Royal Statistical Society. Series B (Methodological), 394-403.

Chkili, W., \& Nguyen, D. K. (2014). Exchange rate movements and stock market returns in a regime-switching environment: Evidence for BRICS countries. Research in International Business and Finance, 31(C), 46-56.

Claessens, S., Dasgupta, S., \& Glen, J. (1995). Return Behavior in Emerging Stock Markets. The World Bank Economic Review, 9(1), 131-151.

Corrado, C. J. (2001). Option pricing based on the generalized lambda distribution. Journal of $\quad$ Futures $\quad$ Markets, 21(3), 213-236. https://doi.org/10.1002/1096-9934(200103)21:3<213::AID-FUT2>3.0.CO;2-H

Cromwell, J. B., Labys, W. C., \& Terraza, M. (1994). Univariate tests for time series models. Sage Publications.

D’Agostino, R. B. (1986). Goodness-of-Fit-Techniques. CRC Press.

Eberlein, E., \& Keller, U. (1995). Hyperbolic distributions in finance. Bernoulli, 1(3), 281-299. https://doi.org/10.3150/bj/1193667819

Eberlein, E., Keller, U., \& Prause, K. (1998). New insights into smile, mispricing, and value at risk: The hyperbolic model*. The Journal of Business, 71(3), 371-405.

Errunza, V. R., \& Padmanabhan, P. (1988). Further evidence on the benefits of portfolio investments in emerging markets. Financial Analysts Journal, 44(4), 76-78.

Fama, E. F., \& MacBeth, J. D. (1973). Risk, Return, and Equilibrium: Empirical Tests. Journal of Political Economy, 81(3), 607-636.

Fisher, R. A. (1922). On the Mathematical Foundations of Theoretical Statistics. Philosophical Transactions of the Royal Society of London A: Mathematical, Physical and 
Engineering Sciences, 222(594-604), 309-368. https://doi.org/10.1098/rsta.1922.0009

Focardi, S. M., \& Fabozzi, F. J. (2004). The Mathematics of Financial Modeling and Investment Management. Hoboken, New Jersey: John Wiley \& Sons, Inc.

Freimer, M., Kollia, G., Mudholkar, G. S., \& Lin, C. T. (1988). A study of the generalized Tukey lambda family. Communications in Statistics-Theory and Methods, 17(10), 3547-3567.

Giot, P., \& Laurent, S. (2004). Modelling daily value-at-risk using realized volatility and ARCH type models. Journal of Empirical Finance, 11(3), 379-398.

Hamilton, J. D. (1989). A new approach to the economic analysis of nonstationary time series and the business cycle. ECONOMETRICA, 57(2), 357-384.

Hansen, L. P. (1982). Large Sample Properties of Generalized Method of Moments Estimators. Econometrica, 50(4), 1029-1054. https://doi.org/10.2307/1912775

Hansen, L. P., Heaton, J., \& Yaron, A. (1996). Finite-Sample Properties of Some Alternative GMM Estimators. Journal of Business \& Economic Statistics, 14(3), 262-280. https://doi.org/10.1080/07350015.1996.10524656

Hassan, M. K., Maroney, N. C., El-Sady, H. M., \& Telfah, A. (2003). Country risk and stock market volatility, predictability, and diversification in the Middle East and Africa. Economic Systems, 27(1), 63-82.

Hearn, B., Piesse, J., \& Strange, R. (2010). Market liquidity and stock size premia in emerging financial markets: The implications for foreign investment. International Business Review, 19(5), 489-501.

Hull, J. C., \& White, A. D. (1998). Value at Risk When Daily Changes in Market Variables are not Normally Distributed. The Journal of Derivatives, 5(3), 9-19. https://doi.org/10.3905/jod.1998.407998

Iacus, S. M. (2011). Option Pricing and Estimation of Financial Models with $R$ (1st ed.). West Sussex, PO19 8SQ, United Kingdom: John Wiley \& Sons, Ltd.

Karian, Z. A., \& Dudewicz, E. J. (1999). Fitting the generalized lambda distribution to data: a method based on percentiles. Communications in Statistics - Simulation and Computation, 28(3), 793-819. https://doi.org/10.1080/03610919908813579

Karian, Z. A., \& Dudewicz, E. J. (2000). Fitting Statistical Distributions: The Generalized Lambda Distribution and Generalized Bootstrap Methods. CRC Press.

Karvanen, J., \& Nuutinen, A. (2008). Characterizing the generalized lambda distribution by L-moments. Computational Statistics \& Data Analysis, 52(4), 1971-1983.

King, R. A. R., \& MacGillivray, H. L. (1999). A Starship Estimation Method for the Generalized Distributions. Australian \& New Zealand Journal of Statistics, 41(3), 353-374. https://doi.org/10.1111/1467-842X.00089

Koudou, A. E., \& Ley, C. (2014). Characterizations of GIG laws: A survey. Probability 
Surveys, 11, 161-176. https://doi.org/10.1214/13-PS227

M. R., H. (2001). A Regime-SWITCHING MODEL OF LONG-TERM STOCK RETURNS. NORTH AMERICAN ACTUARIAL JOURNAL, 5(2).

Myung, I. J. (2003). Tutorial on maximum likelihood estimation. Journal of Mathematical Psychology, 47(1), 90-100.

Newey, W., \& West, K. (1987). A Simple, Positive Semi-definite, Heteroskedasticity and Autocorrelation Consistent Covariance Matrix. Econometrica, 55(3), 703-08.

Owen, A. B. (1988). Empirical likelihood ratio confidence intervals for a single functional. Biometrika, 75(2), 237-249. https://doi.org/10.1093/biomet/75.2.237

Öztürk, A., \& Dale, R. F. (1982). A Study of Fitting the Generalized Lambda Distribution to Solar Radiation Data. Journal of Applied Meteorology, 21(7), 995-1004. https://doi.org/10.1175/1520-0450(1982)021<0995:ASOFTG>2.0.CO;2

Öztürk, A., \& Dale, R. F. (1985). Least squares estimation of the parameters of the generalized lambda distribution. Technometrics, 27(1), 81-84.

Pearson, K. (1894). Contributions to the Mathematical Theory of Evolution. Philosophical Transactions of the Royal Society of London A: Mathematical, Physical and Engineering Sciences, 185, 71-110. https://doi.org/10.1098/rsta.1894.0003

Pearson, K. (1936). Method of Moments and Method of Maximum Likelihood. Biometrika, 28(1/2), 34-59. https://doi.org/10.2307/2334123

Pfaff, B. (2013). Financial Risk Modelling and Portfolio Optimization with R. West Sussex, PO19 8SQ, United Kingdom: John Wiley \& Sons Ltd.

Prause, K. (1997). Modelling financial data using generalized hyperbolic distributions. fDM Preprint, $48 . \quad$ Retrieved from http://citeseerx.ist.psu.edu/viewdoc/download?doi=10.1.1.416.1199\&rep=rep1\&type=pdf

Prause, K. (1999). The generalized hyperbolic model: Estimation, financial derivatives, and risk measures. Citeseer. Retrieved from http://citeseerx.ist.psu.edu/viewdoc/download?doi=10.1.1.382.9958\&rep=rep1\&type=pdf

Ramberg, J. S., Dudewicz, E. J., Tadikamalla, P. R., \& Mykytka, E. F. (1979). A probability distribution and its uses in fitting data. Technometrics, 21(2), 201-214.

Ramberg, J. S., \& Schmeister, B. W. (1974). An approximate method for generating asymmetric random variables. Communication of the ACM, 17, 78-82.

Ranneby, B. (1984). The maximum spacing method. An estimation method related to the maximum likelihood method. Scandinavian Journal of Statistics, 93-112.

R Core Team. (2016). R: A language and environment for statistical computing. $R$ Foundation for Statistical Computing. Vienna, Austria. Retrieved from URL http://www.R-project.org/ 


\section{Macrothink}

Asian Journal of Finance \& Accounting

ISSN 1946-052X

2017, Vol. 9, No. 1

Sørensen, M. (2006). On the size distribution of sand. Unpublished Paper,Department of Applied Mathematics and Statistics University of Copenhagen Universitetsparken 5, DK-2100 Copenhagen Ø, Denmark, 10.

Stock, J., Wright, J., \& Yogo, M. (2002). A Survey of Weak Instruments and Weak Identification in Generalized Method of Moments. Journal of Business \& Economic Statistics, 20, 518-529.

Su, S. (2005). A Discretized Approach to Flexibly Fit Generalized Lambda Distributions to Data. Journal of Modern Applied Statistical Methods, 4(2). Retrieved from http://digitalcommons.wayne.edu/jmasm/vol4/iss2/7

$\mathrm{Su}$, S. (2007a). Fitting single and mixture of generalized lambda distributions to data via discretized and maximum likelihood methods: GLDEX in R. Journal of Statistical Software, 21(9), 1-17.

Su, S. (2007b). Numerical Maximum Log Likelihood Estimation for Generalized Lambda Distributions. Comput. Stat. Data Anal., 51(8), 3983-3998. https://doi.org/10.1016/j.csda.2006.06.008

Tarsitano, A. (2004). Fitting the generalized lambda distribution to income data (pp. 1861-1867). Presented at the In COMPSTAT'2004 Symposium.

Tsay, R. S. (2010). Analysis of Financial Time Series (Third Edition). Hoboken, New Jersey: John Wiley \& Sons, Inc.

Van Norden, S., \& Schaller, H. (1993). The Predictability of Stock Market Regime: Evidence from the Toronto Stock Exchange. The Review of Economics and Statistics, 75(3), 505-10.

Wooldridge, J. M. (2001). Applications of Generalized Method of Moments Estimation. The Journal of Economic Perspectives, 15(4), 87-100. 